nPak. J. Agri. Sci., Vol. 53(2), 291-301; 2016

ISSN (Print) 0552-9034, ISSN (Online) 2076-0906

DOI: $10.21162 / P A K J A S / 16.3143$

http://www.pakjas.com.pk

\title{
AGRONOMIC TRAITS AND CYTOGENETIC EVALUATION OF NEWLY DEVELOPED AUTOTETRAPLOID RICE LINE
}

\author{
Haibin Guo", Muhammad Qasim Shahid", Jian Zhao", Yajuan Li, Lan Wang and \\ Xiangdong Liu*
}

\author{
State Key Laboratory for Conservation and Utilization of Subtropical Agro-Bioresources, South China Agricultural \\ University, Guangzhou 510642, China. \\ *Corresponding author's e-mail: xdliu@scau.edu.cn \\ IIThese authors contributed equally to this work.
}

\begin{abstract}
Autotetraploid rice is an important germplasm and has a great scope to increase rice yield but low seed set is a major encumbrance in its utilization. The present study was planned to observe the cytogenetic and agronomic characteristics of newly developed highly fertile autotetraploid rice line (1162). Agronomic traits, male and female gametophytes of newly developed autotetraploid rice (1162) and their hybrids showed better performance as compared to its parents and other autotetraploid rice lines. The mean of panicle length, number of grains per panicle, filled grains per panicle, 1000-grain weight, grain length, grain width, and seed set were $25.04 \mathrm{~cm}, 103.38$ grains, 83.56 grains, $43.44 \mathrm{~g}, 9.54 \mathrm{~mm}, 4.04 \mathrm{~mm}$ and $81.58 \%$, respectively. The meiotic analysis showed that the mean meiotic configurations of 1162 at diakinesis were $(0.13 \pm 0.05) \mathrm{I}+$ $(4.89 \pm 0.26)$ II $+(0.02 \pm 0.00)$ III $+(9.45 \pm 0.13)$ IV. The frequency of trivalent and univalent in new line was lower than the autotetraploid lines (control) at diakinesis and metaphase I, and the configuration of chromosome in 1162 was more symmetrical, which was in favor of $2 / 2$ segregation of homologous chromosome, although higher number of quadrivalents were found than parents. Heterosis of hybrids crossed with 1162 was not only positive but also attained highly significant levels for important quantitative traits. The results showed that high fertility can be inherited and have important applications and research value.
\end{abstract}

Keywords: Agronomic traits, autotetraploid rice, Oryza sativa, polyploidy, seed setting.

\section{INTRODUCTION}

Rice is one of the most important crops in China and whole world, which is the model cereal organism because of its small genome. The yield of rice is related to the food security of China and world. So, how to improve the yield and resistance of rice have attracted the attention of breeders. One of the most important aspects of breeding methods to raise rice production is the utilization of the heterosis at the diploid level. The rice yield has been obviously increased by the use of the heterosis, which improve living standards of the people. However, as the climate warms up, the environment worsens and the damage of the diseases and pests increases, the methods of rice breeding are now facing challenges such as: the productivity of rice has been stagnant and the resistance level is not increased significantly. The breeding of autotetraploid rice seems to be an approach worth exploring, because of its gigantic characteristics (Cai et al., 2001; Shahid et al., 2011 and 2013a). Autotetraploid rice is a new germplasm which is obtained by chromosome doubling through colchicine treatment. Autotetraploid rice showed higher genetic variation, greater stability across varying environments, resistant to lodging, higher hybrid vigor, greater grain length and width and resistant to insect pest and diseases than diploid rice (Song and Zhang, 1992; Luan et al., 2008; Tu et al., 2007; Shahid et al., 2011, 2012; Wu et al., 2013). However, Low seed set is the major barrier in autotetraploid rice breeding (He et al., 2011a; Shahid et al., 2013a). There are many reasons for low seed setting of autotetraploid rice, such as pollen and embryo sac abortion (Huang et al., 1999; Zhang et al., 2003; Guo et al., 2006; Hu et al., 2010; Shahid et al., 2010), abnormal chromosome behavior and microtubule distribution pattern, and differentially expressed genes and miRNAs (He et al, 2011a,b; Wu et al., 2014, 2015; Li et al., 2016). Indicajaponica autotetraploid rice hybrids have shown strong yield potential, which would provide a new way to the rice breeding (Shahid et al., 2012). But the poor fertility or low seed set is the inherent problem which has limited the yield advantages of autotetraploid hybrid rice breeding. In order to overcome this problem, through continuous hard work for 20 years, we have developed a large number of autotetraploid rice lines after chromosome doubling with varying seed set. Some new autotetraploid rice lines with high fertility were developed in our lab from 2007. Therefore, the present study was planned to 1) investigate the cytogenetic and agronomic characteristics of newly developed high fertility autotetraploid rice line, 2) and to observe the hybrid vigor by 
crossing with other 42 autotetraploid rice lines. These lines could provide the important germplasm for autotetraploid rice breeding, and practical and theoretical basis for heterosis studies, and for commercial utilization of autotetraploid rice.

\section{MATERIALS AND METHODS}

Plant materials: High fertility autotetraploid rice line "1162" was developed in our lab in 2010 by crossing autotetraploid Jackson-4x and 96025. Jackson-4x, which was kindly donated by Prof. YQ Li, South China Botanical Garden, Chinese academy of sciences (SCBU-CAS), was obtained by chromosome doubling through colchicine treatment from an American rice variety, Jackson-2x. 96025 is an autotetraploid rice line, and kindly provided by Prof. YH Zhang, Chinese Academy of Agricultural Sciences. 1162 and its parents were used for investigating chromosome behavior during meiosis in the present study. Forty two hybrids were developed by crossing 1162 with 42 autotetraploid rice lines to investigate mid-parent heterosis and better-parent heterosis. Pollen and embryo sac fertility of 1162 , its parents, and $1162 \times$ Jingxian 89-4x, 1162×4001-1-4x 1162xYuhei1-4x and 1162×IR36-4x were observed. All these materials were planted at the farm of South China Agricultural University.

Chromosome behavior observation: Inflorescences were collected from shoots of rice plants with 1-4 cm between their flag leaf cushion and second-to-last leaf cushion, and fixed in Carnoy solution (ethanol : acetic acid=3:1) for at least $24 \mathrm{~h}$. The samples were then stored in $70 \%$ ethanol at $4^{\circ} \mathrm{C}$. Anthers were separated from the floret using forceps and dissecting needle and placed in a small drop of $1 \%$ carmine acetate. After 2-3 min, glass slide was covered with a slide cover and examined under microscope (Olympus CX31). The numbers of the quadrivalent, bivalent, trivalent and univalent were also observed according to He et al. (2011b).
Investigation of pollen and embryo sac fertility and seed setting percentage: About five mature spikelets were collected from various panicles of the plant to investigate the pollen fertility, and then fixed in Carnoy solution for $24 \mathrm{~h}$. Potassium Iodide solution $\left(\mathrm{I}_{2}-\mathrm{KI}, 1 \%\right)$ was used to stain the spikelets and observed under microscope. Pollen fertility was divided into four categories i.e., normal pollens, stained abortive pollens, spherical abortive pollens and typical abortive pollens (Shahid et al., 2013b).

Whole-mount eosin B-staining confocal laser scanning microscopy (WE-CLSM) was used to observe embryo-sac structure. Embryo sac fertility was investigated according to Shahid et al. (2010) and seed setting was counted according to the method of Shahid et al. (2013b).

The amount of heterosis or hybrid vigor $(H)$ was evaluated using two measurements: (1) mid-parent heterosis $\left(H_{P M}\right)$ was calculated using the equation: $H=\left(F_{1}-\left(P_{1}+P_{2}\right) / 2\right) /\left(P_{1}+P_{2}\right) / 2$ (where $P_{1}$ and $P_{2}$ are the means of the corresponding parents), and (2) better-parent heterosis using the following equation $\left(H_{P B}\right): H=\left(F_{1}-P_{b}\right) / P_{b}$ (where $P_{b}$ represents the value of the better-parent).

\section{RESULTS}

Performance of main agronomic traits in 1162: The means of ten agronomic traits of 1162 and its parents were assessed by analysis of variance (Table 1 ). The results indicated that six traits of 1162, including plant height, the number of filled grains per panicle, seed setting, 1000-grain weight, grain width and grain length-width ratio, were significantly $(p<0.01)$ higher than parents. The number of filled grains per panicle, seed setting and 1000-grain weight were closely related with yield. Number of grains per panicle and grain length of 1162 significantly increased as compared to its parents, while there was non-significant difference in the

Table 1. Analysis of variance for agronomic characters of 1162 and its parents.

\begin{tabular}{lcccc}
\hline Agronomic characters & $\mathbf{9 6 0 2 5}$ & Jackson-4x & $\mathbf{1 1 6 2}$ & $\boldsymbol{F}$ value \\
\hline $\mathrm{PH} / \mathrm{cm}$ & $84.86 \pm 1.63 \mathrm{c}$ & $93.32 \pm 0.81 \mathrm{~b}$ & $120.78 \pm 2.78 \mathrm{a}$ & $95.67 * *$ \\
$\mathrm{NP}$ & $4.00 \pm 0.32 \mathrm{c}$ & $3.00 \pm 0.32 \mathrm{c}$ & $4.00 \pm 0.45 \mathrm{c}$ & 2.50 \\
$\mathrm{PL} / \mathrm{cm}$ & $26.64 \pm 1.02 \mathrm{c}$ & $25.86 \pm 0.71 \mathrm{c}$ & $25.34 \pm 0.66 \mathrm{c}$ & 0.65 \\
NGP & $91.56 \pm 3.98 \mathrm{~b}$ & $114.94 \pm 6.54 \mathrm{a}$ & $106.98 \pm 6.05 \mathrm{ab}$ & $4.45 *$ \\
NFGP & $49.44 \pm 4.01 \mathrm{~b}$ & $32.14 \pm 1.90 \mathrm{c}$ & $89.80 \pm 6.33 \mathrm{a}$ & $43.99 * *$ \\
SS $(\%)$ & $53.82 \pm 2.89 \mathrm{~b}$ & $28.03 \pm 1.15 \mathrm{c}$ & $83.74 \pm 2.28 \mathrm{a}$ & $156.83 * *$ \\
TGW/g & $43.18 \pm 0.83 \mathrm{~b}$ & $25.18 \pm 0.87 \mathrm{c}$ & $43.37 \pm 0.75 \mathrm{~b}$ & $162.58 * *$ \\
GL/mm & $9.24 \pm 0.14 \mathrm{c}$ & $10.52 \pm 0.24 \mathrm{~b}$ & $9.46 \pm 0.14 \mathrm{c}$ & $14.54 *$ \\
GW/mm & $3.34 \pm 0.09 \mathrm{~b}$ & $2.96 \pm 0.068 \mathrm{c}$ & $3.96 \pm 0.14 \mathrm{a}$ & $24.04 * *$ \\
L/W & $2.78 \pm 0.11 \mathrm{~b}$ & $3.56 \pm 0.07 \mathrm{a}$ & $2.40 \pm 0.11 \mathrm{c}$ & $38.34 * *$ \\
\hline
\end{tabular}

$\mathrm{PH}=$ Plant Height, NP=Number of Panicles, $\mathrm{PL}=$ Panicle Length, NGP= Number of Grains per Plant, NFGP= Number of Filled Grains per Panicle, SS=Seed Setting, TGW=1000-Grain Weight, GL=Grain Length, GW=Grain Width, L/W=Grain Length-Width Ratio.

${ }^{*},{ }^{* *}$ Significantly different from zero at $\mathrm{p}<0.05$ and $\mathrm{p}<0.01$, respectively 
number of panicles and panicle length between 1162 and parents. 1162 also showed higher seed setting $(83.74 \%)$ than its parents, which reached at the level of diploid rice (Fig. 1).

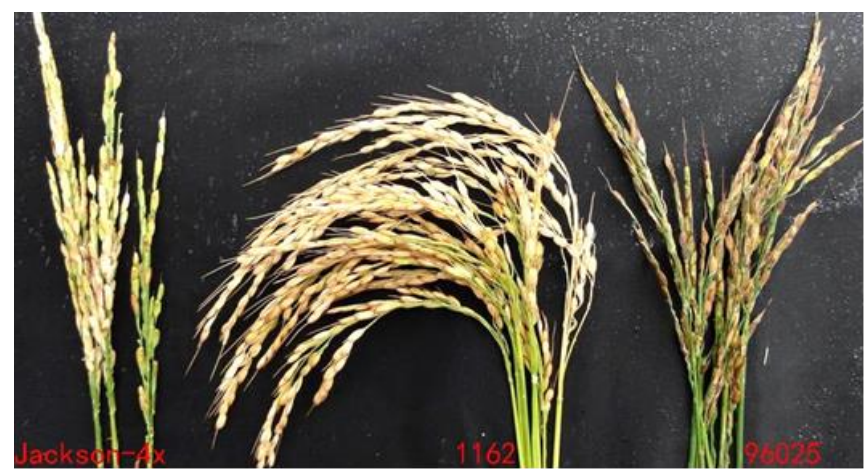

Figure 1. Panicles of 1162 and its parents.

Chromosome configuration during pollen mother cell meiosis in high fertile autotetraploid rice: The high fertile autotetraploid rice and its parents were used to observe pollen mother cell (PMC) meiosis and chromosome behavior. The results showed that meiosis division of high fertility autotetraploid rice line and its parents were consistent with diploid rice. Meiosis process include: prophase I, metaphase I, anaphase I, telophase I, prophase II, metaphase II, anaphase II and telophase II. Prophase I could be subdivided into leptotene (Plate I-1, II-1), zygotene (Plate I-2, II-2), pachytene (Plate I- 3), diplotene (Plate I-4, II-3) and diakinesis (Plate I-5, II-4). Diakinesis is the best stage to identify the configuration and number of chromosomes. There were highly significant differences between newly developed autotetraploid rice line and their parents for chromosome configuration at diakinesis and following results were obtained.

96025: $2 \mathrm{n}=48=(0.27 \pm 0.08) \mathrm{I}+(6.26 \pm 0.38) \mathrm{II}+(0.03 \pm 0.02)$ $\mathrm{III}+(8.59 \pm 0.20) \mathrm{IV}$

Jackson-4x: $2 \mathrm{n}=48=(0.39 \pm 0.08) \quad \mathrm{I}+(8.93 \pm 0.41) \mathrm{II}+$ $(0.12 \pm 0.04) \mathrm{III}+(7.02 \pm 0.20) \mathrm{IV}$

1162: $2 \mathrm{n}=48=(0.13 \pm 0.05) \mathrm{I}+(4.89 \pm 0.26) \mathrm{II}+(0.02 \pm 0.00) \mathrm{III}$

$+(9.45 \pm 0.13) \mathrm{IV}$
There were highly significant differences between parents and 1162 for the number of quadrivalents. The numbers of quadrivalents per cell ranged from 3-12, the average numbers of quadrivalents per cell were over 7. New line 1162 had the highest number of quadrivalents per cell (9.45), whereas female parent (Jackson-4x) had the lowest number of quadrivalents per cell (7.02). The main types of quadrivalents were: ring-shape (Plate II-4), double ring-shape (Plate II-4), chain-shape (Plate I-5), frying pan-shape, Y shape, OK shape (Plate I-5), X shape and -O- shape. Ring and double ring shapes were the most frequent configurations of quadrivalents. 1162 and Jackson-4x had the highest (4.28) and the lowest (2.91) number of double rings quadrivalents, respectively (Table 2).

There were different number of bivalents (ranging from 5 to 9), trivalents, univalents and multivalents in 1162 and its parents (Table 2). Jackson-4x had the highest number of bivalents per cell (8.93), while 1162 had the lowest number of bivalents per cell (4.89). The types of bivalents in autotetraploid rice were rod and ring shape as in diploid rice. There were more ring bivalents than rod bivalents in autotetraploid rice, and a few quadrivalents changed into trivalents and univalents. The main types of trivalents were chain and frying pan shape. Average frequency of trivalents was less than 1 in all autotetraploid rice lines. Jackson- $4 \mathrm{x}$ with the lowest seed setting had the highest number of univalents and multivalents, while the new line has significantly lower number of univalents and multivalents than Jackson-4x and 96025.

Metaphase I: Most of the chromosomes arranged at the equator of the spindle (Plate I -6, II-5). Chromosome straggling (Plate III-1) and abnormal spindle (Plate III-2) were also observed during this stage. The maximum percentage of PMCs with chromosome straggling was $33.08 \%$ in Jackson-4x, while the minimum percentage of chromosome straggling (18.40\%) was found in 1162 .

Anaphase I: Homologous chromosomes were separated towards opposite poles of the cell (Plate I-7, II-6). The main types of abnormalities include: chromosome straggling (Plate III-3), chromosome bridge (Plate III-4), abnormal spindle and asymmetric division (Plate III-5).

Table 2. Chromosome configurations at diakinesis in autotetraploid rice lines.

\begin{tabular}{|c|c|c|c|c|c|c|c|c|c|c|c|c|c|c|c|c|}
\hline \multirow[t]{2}{*}{ Materials } & \multirow[t]{2}{*}{$\mathbf{N}$} & \multicolumn{7}{|c|}{ Quadrivalents per cell } & \multicolumn{3}{|c|}{ Trivalents per cell } & \multicolumn{3}{|c|}{ Bivalents per cell } & \multirow{2}{*}{$\begin{array}{c}\text { Uni- } \\
\text { valent } \\
\text { per cell }\end{array}$} & \multirow{2}{*}{$\begin{array}{l}\text { Multi- } \\
\text { valents } \\
\text { per cell }\end{array}$} \\
\hline & & Ring & Chain & F.pan & $\mathbf{Y}$ & D rings & Others & Total & Chain & F.pan & Total & Rod & Ring & Total & & \\
\hline 96025 & 93 & $\begin{array}{c}3.42 \\
\pm 0.18\end{array}$ & $\begin{array}{c}0.91 \\
\pm 0.09\end{array}$ & $\begin{array}{c}0.37 \\
\pm 0.08\end{array}$ & $\begin{array}{c}0.01 \\
\pm 0.01\end{array}$ & $\begin{array}{c}3.62 \\
\pm 0.23\end{array}$ & $\begin{array}{c}0.42 \\
\pm 0.08\end{array}$ & $\begin{array}{c}8.59 \\
\pm 0.20\end{array}$ & $\begin{array}{c}0.03 \\
\pm 0.02\end{array}$ & 0.00 & $\begin{array}{c}0.03 \\
\pm 0.02\end{array}$ & $\begin{array}{c}1.79 \\
\pm 0.17\end{array}$ & $\begin{array}{c}4.71 \\
\pm 0.34\end{array}$ & $\begin{array}{c}6.26 \\
\pm 0.38\end{array}$ & $\begin{array}{c}0.27 \\
\pm 0.08\end{array}$ & 0.00 \\
\hline Jackson- $4 \mathrm{x}$ & 85 & $\begin{array}{c}2.99 \\
\pm 0.15\end{array}$ & $\begin{array}{c}0.66 \\
\pm 0.09\end{array}$ & $\begin{array}{c}0.39 \\
\pm 0.07\end{array}$ & $\begin{array}{c}0.02 \\
\pm 0.02\end{array}$ & $\begin{array}{c}2.91 \\
\pm 0.16\end{array}$ & $\begin{array}{c}0.26 \\
\pm 0.05\end{array}$ & $\begin{array}{c}7.02 \\
\pm 0.20\end{array}$ & $\begin{array}{c}0.02 \\
\pm 0.02\end{array}$ & $\begin{array}{c}0.09 \\
\pm 0.03\end{array}$ & $\begin{array}{c}0.12 \\
\pm 0.04\end{array}$ & $\begin{array}{c}0.64 \\
\pm 0.09\end{array}$ & $\begin{array}{c}8.31 \\
\pm 0.39\end{array}$ & $\begin{array}{c}8.93 \\
\pm 0.41\end{array}$ & $\begin{array}{c}0.39 \\
\pm 0.08\end{array}$ & $\begin{array}{c}0.16 \\
\pm 0.05\end{array}$ \\
\hline 1162 & 105 & $\begin{array}{c}3.87 \\
\pm 0.17\end{array}$ & $\begin{array}{c}0.86 \\
\pm 0.09\end{array}$ & $\begin{array}{c}0.19 \\
\pm 0.05\end{array}$ & $\begin{array}{c}0.04 \\
\pm 0.02\end{array}$ & $\begin{array}{c}4.28 \\
\pm 0.19\end{array}$ & $\begin{array}{c}0.27 \\
\pm 0.05\end{array}$ & $\begin{array}{c}9.45 \\
\pm 0.13\end{array}$ & $\begin{array}{c}0.02 \\
\pm 0.01\end{array}$ & 0.00 & $\begin{array}{c}0.02 \\
\pm 0.00\end{array}$ & $\begin{array}{c}0.58 \\
\pm 0.09\end{array}$ & $\begin{array}{c}4.30 \\
\pm 0.24\end{array}$ & $\begin{array}{c}4.89 \\
\pm 0.26\end{array}$ & $\begin{array}{c}0.13 \\
\pm 0.05\end{array}$ & 0.00 \\
\hline
\end{tabular}

$\mathrm{N}=$ No. of cells; Ring=Ring shape; Chain=Chain shape; F.pan=Frying pan shape; Y=Y shape; D rings=Double rings shape. 


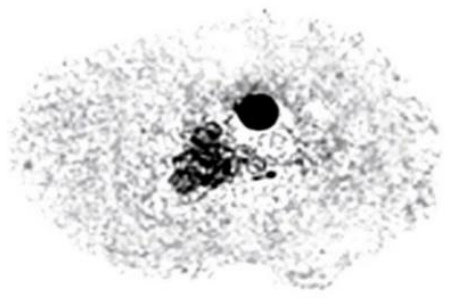

1
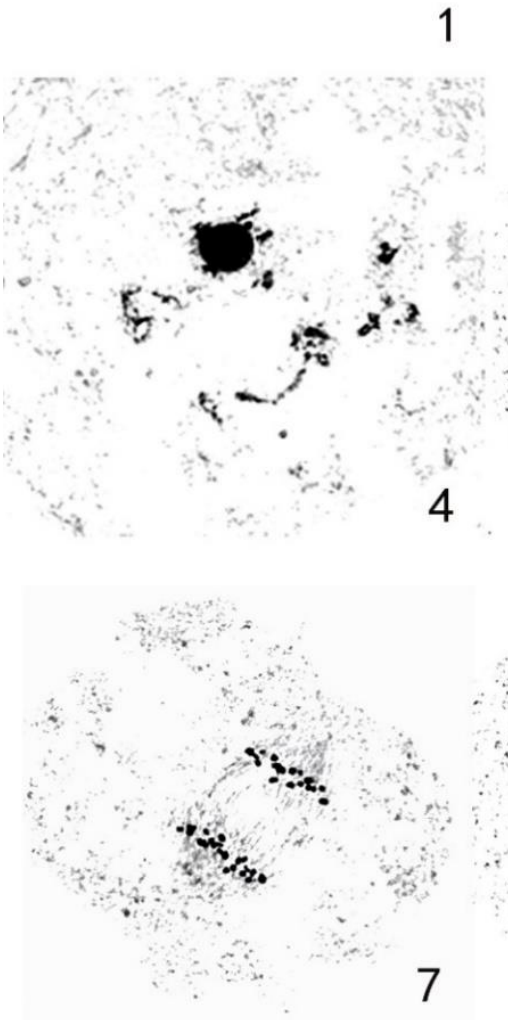

7
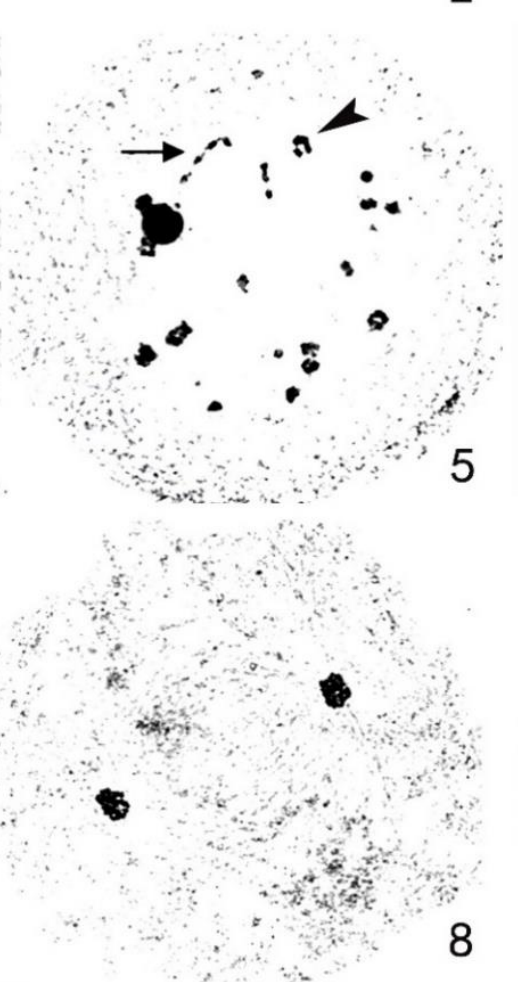
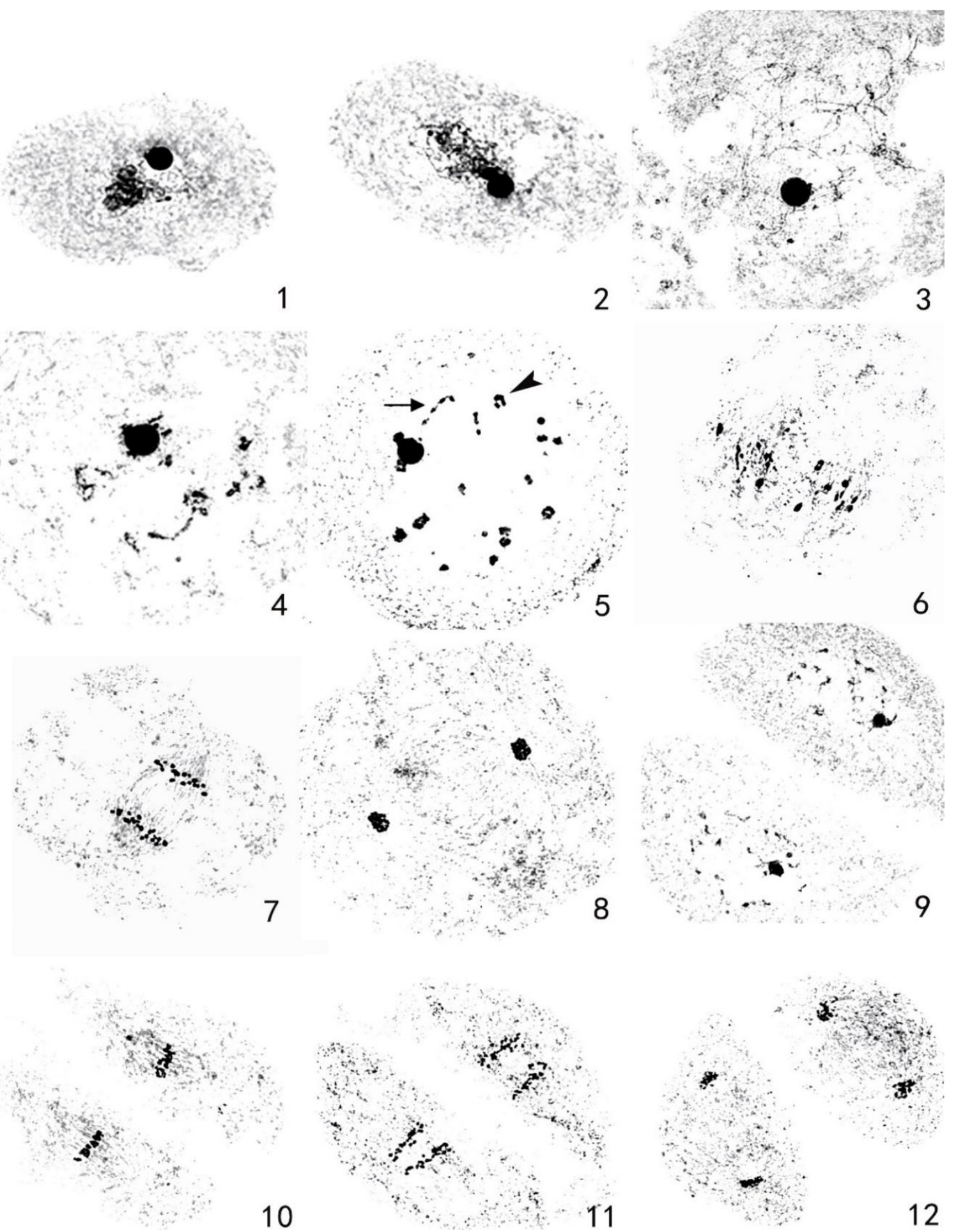

Plate I. Chromosome behavior of PMCs in Jackson-4x, $\times 1000$.

1. Leptotene; 2. Zygotene; 3. Pachytene; 4. Diplotene; 5. Diakinesis, VII +7 IV +6 II + I, chain-shape quadrivalent (arrow head), OK shape quadrivalent (arrow); 6. Metaphase I, 7 IV +10 II; 7. Anaphase I; 8. Telophase I; 9. Prophase II; 10. Metaphase II; 11. Anaphase II; 12. Telophase II 

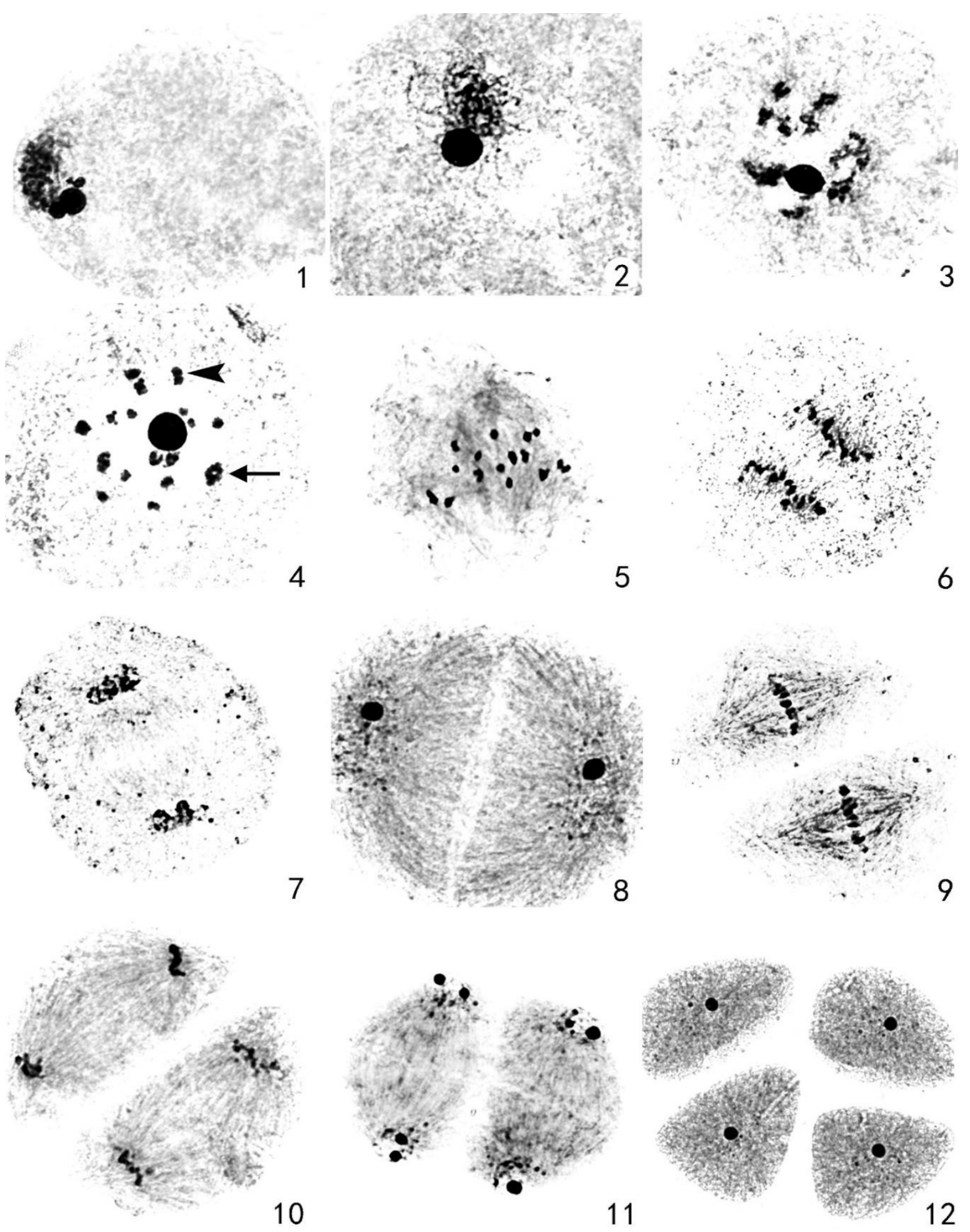

Plate II. Chromosome behavior of PMCs in $1162, \times 1600$.

1. Leptotene; 2. Zygotene; 3. Diplotene; 4.Diakinesis, 8 IV+7 II+2 I, ring-shape quadrivalent (arrow head), double ring-shape quadrivalent (arrow); 5. Metaphase I, 11 IV+2 II; 6. Anaphase I; 7. Telophase I; 8. Prophase II; 9. Metaphase II; 10. Anaphase II; 11. Telophase II; 12. Tetrads 

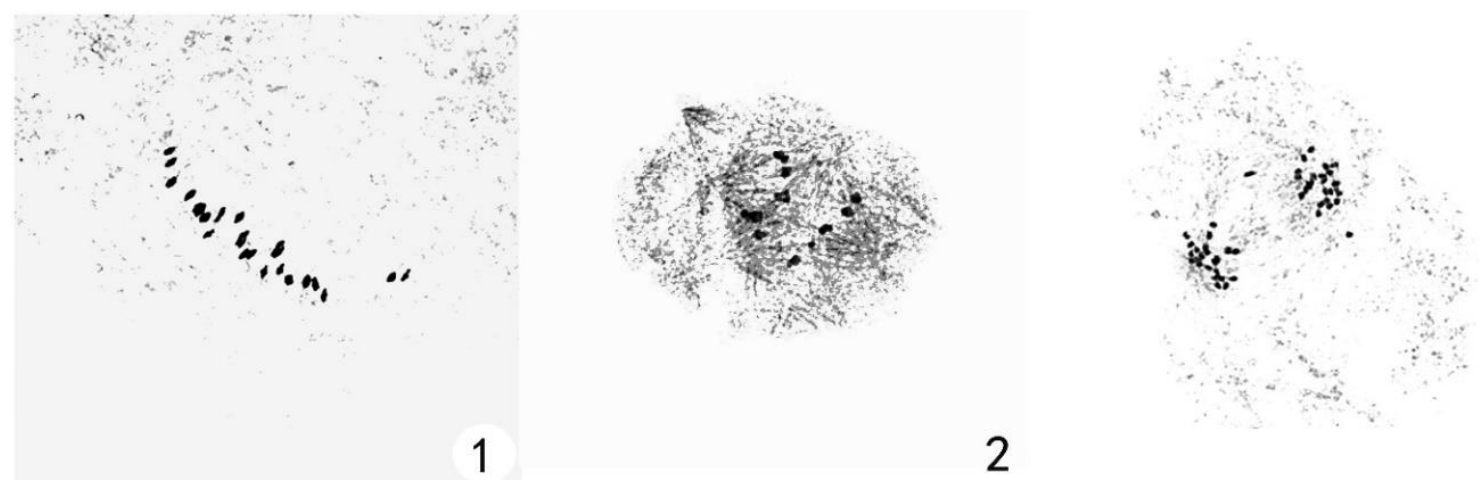

2

3
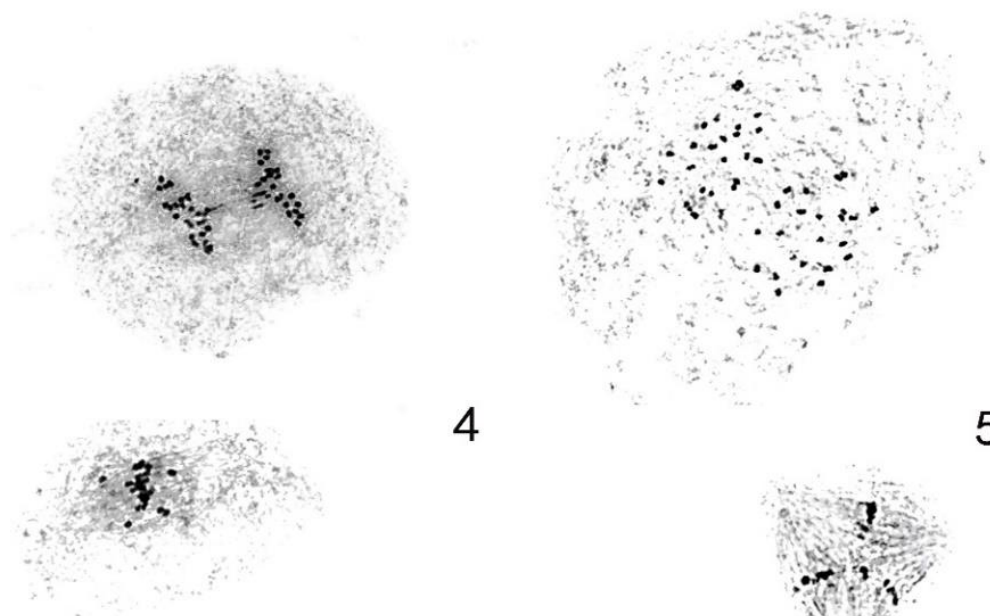

4

5

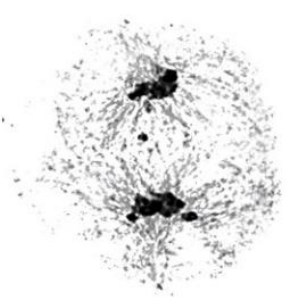

$5 \quad 6$
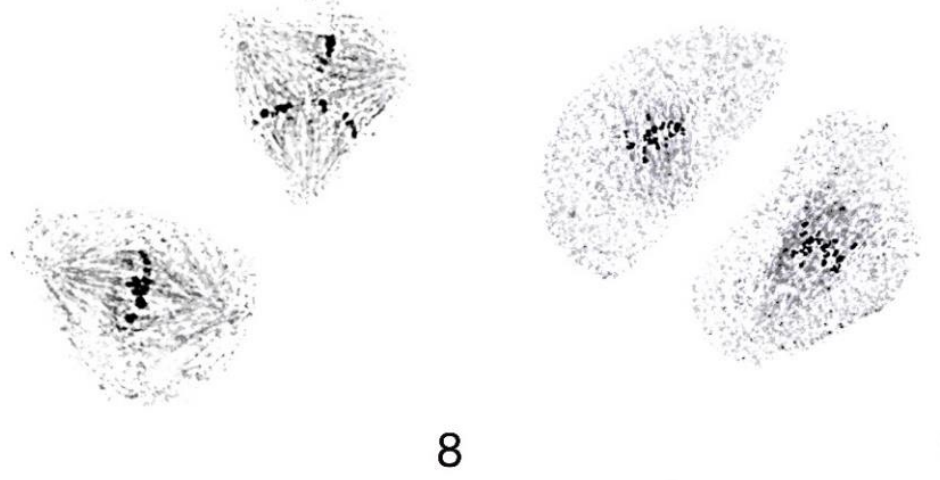

8

9
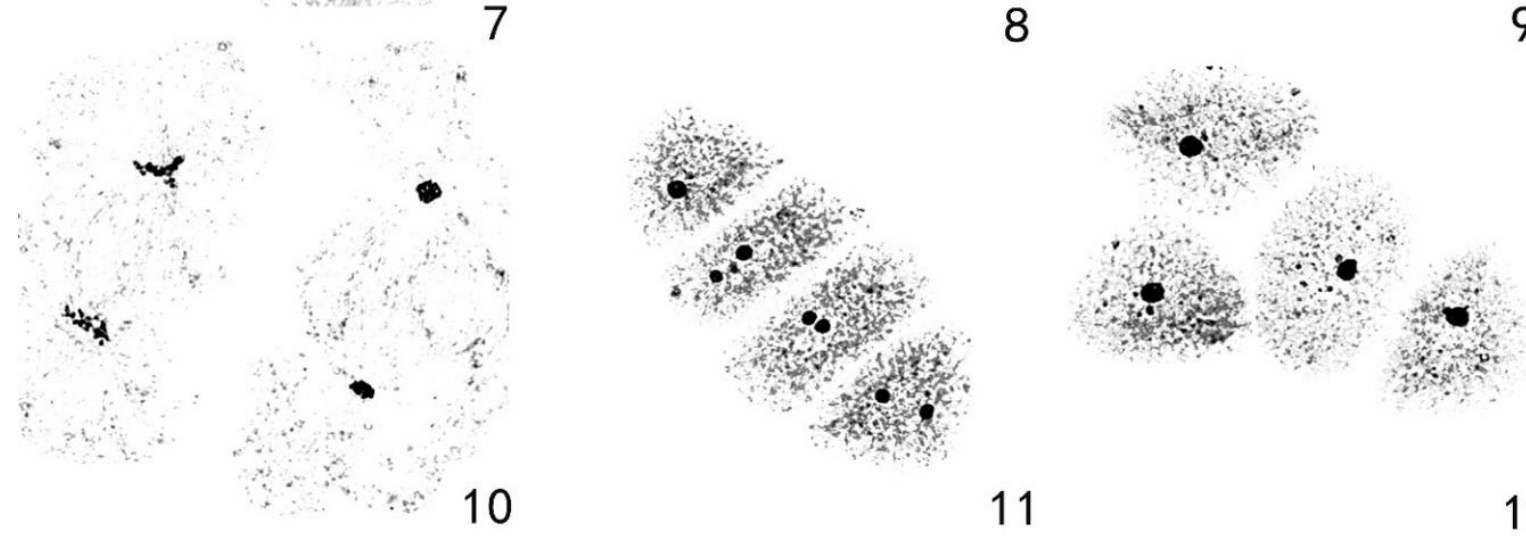

11

12

Plate III. Abnormal chromosome behavior of PMCs in autotetraploid rice, $\times \mathbf{1 0 0 0}$.

1. Metaphase I, straggling (Jackson-4x); 2. Metaphase I, multipolar spindle (96025); 3. Anaphase I, Straggling (Jackson-4x); 4. Anaphase I, chromosome bridge (96025) 5. Anaphase I (Jackson-4x); 6. Telophase I, Straggling (96025); 7. Metaphase II, straggling (Jackson-4x): 8. Metaphase II, triad (96025) 9. Metaphase II, T spindle (Jackson-4x); 10. asynchronous division, (Jackson-4x); 11. Telophase II, linear tetrad (96025); 12. Telophase II, T tetrad (96025) 
Table 3. Frequency of abnormal chromosome behaviors during meiosis I of autotetraploid rice lines.

\begin{tabular}{|c|c|c|c|c|c|c|c|c|c|c|c|c|c|}
\hline \multirow[t]{2}{*}{ Name } & \multicolumn{4}{|c|}{ Metaphase I } & \multicolumn{4}{|c|}{ Anaphase I } & \multicolumn{5}{|c|}{ Telophase I } \\
\hline & $\mathbf{N}$ & $\begin{array}{c}\text { Normal } \\
(\%)\end{array}$ & $\begin{array}{l}\text { Lag } \\
(\%)\end{array}$ & $\begin{array}{c}\text { Multipolar } \\
\text { spindle (\%) }\end{array}$ & $\mathbf{N}$ & $\begin{array}{c}\text { Normal } \\
(\%)\end{array}$ & $\begin{array}{c}\text { Strag } \\
(\%)\end{array}$ & $\begin{array}{c}\text { Bridge } \\
(\%)\end{array}$ & $\mathbf{N}$ & $\begin{array}{c}\text { Normal } \\
(\%)\end{array}$ & $\begin{array}{c}\text { Strag } \\
(\%)\end{array}$ & $\begin{array}{c}\text { Triad } \\
(\%)\end{array}$ & $\begin{array}{c}\text { Micronucleus } \\
(\%)\end{array}$ \\
\hline 96025 & 203 & 77.83 & 15.76 & 6.40 & 75 & 81.33 & 17.33 & 1.33 & 101 & 90.10 & 9.90 & 0.00 & 0.00 \\
\hline Jackson-4x & 130 & 66.92 & 33.08 & 0.00 & 82 & 86.59 & 13.41 & 0.00 & 118 & 80.51 & 16.10 & 0.00 & 3.39 \\
\hline 1162 & 163 & 71.78 & 18.40 & 9.82 & 162 & 87.65 & 12.35 & 0.00 & 118 & 91.53 & 5.08 & 1.69 & 1.69 \\
\hline
\end{tabular}

$\mathrm{N}=$ Number of cells observed; Lag=Lagging; Strag=Straggling

Table 4. Frequency of abnormal chromosome behaviors at metaphase II of autotetraploid rice lines.

\begin{tabular}{lccccccccc}
\hline Name & $\mathbf{N}$ & $\begin{array}{c}\text { Normal } \\
(\boldsymbol{\%})\end{array}$ & $\begin{array}{c}\text { Lag } \\
(\boldsymbol{\%})\end{array}$ & $\begin{array}{c}\text { Tripolar } \\
\text { spindle (\%) }\end{array}$ & $\begin{array}{c}\text { V spindle } \\
(\boldsymbol{\%})\end{array}$ & $\begin{array}{c}\text { T spindle } \\
(\boldsymbol{\%})\end{array}$ & $\begin{array}{c}\text { I spindle } \\
(\boldsymbol{\%})\end{array}$ & $\begin{array}{c}\text { Chaos (\%) } \\
\text { Out of } \\
\text { step (\%) }\end{array}$ \\
\hline 96025 & 74 & 60.81 & 2.70 & 9.46 & 10.81 & 8.11 & 2.70 & 5.41 & 0.00 \\
Jackson-4x & 150 & 55.33 & 24.67 & 0.00 & 8.00 & 2.00 & 0.00 & 0.00 & 10.00 \\
1162 & 124 & 62.10 & 15.32 & 8.87 & 0.00 & 8.06 & 0.00 & 5.65 & 0.00 \\
\hline
\end{tabular}

$\mathrm{N}=$ Number of cells observed; Lag=Lagging

Table 5. Frequency of abnormal chromosome behaviors at anaphase II of autotetraploid rice lines.

\begin{tabular}{lcccccc}
\hline Name & $\mathbf{N}$ & Normal (\%) & Strag (\%) & Triad (\%) & Chaos (\%) & Out of step (\%) \\
\hline 96025 & 27 & 40.74 & 7.41 & 0.00 & 3.70 & 48.15 \\
Jackson-4x & 87 & 37.93 & 12.64 & 0.00 & 0.00 & 49.42 \\
1162 & 57 & 54.39 & 1.75 & 7.02 & 0.00 & 36.85 \\
\hline
\end{tabular}

$\mathrm{N}=$ Number of cells observed; Strag=Straggling

Table 6. Frequency of abnormal chromosome behaviors at telophase II of autotetraploid rice lines.

\begin{tabular}{lcccccccc}
\hline Name & $\mathbf{N}$ & Normal/\% & Strag/\% & Abnormal tetrad/\% & Pentads/\% & Out of step/\% & Polyad/\% & Micronucleus/\% \\
\hline 96025 & 152 & 84.87 & 1.32 & 13.16 & 0.66 & 0.00 & 0.00 & 0.00 \\
Jackson-4x & 84 & 73.81 & 8.33 & 0.00 & 0.00 & 4.76 & 0.00 & 13.10 \\
1162 & 121 & 86.78 & 0.00 & 3.31 & 3.31 & 2.48 & 4.13 & 0.00 \\
\hline
\end{tabular}

$\mathrm{N}=$ Number of cells observed; Strag=Straggling

The frequency of chromosome straggling was ranged from 12.35 to $17.33 \%$. The frequency of normal cell in new line was significantly higher than parents (Table 3). Straggled chromosomes were also observed at Telophase I, but their frequency was less in new line than its parents. Prophase II, fission begins with division of nucleus, followed by cytoplasm division, and then a dyad was formed (Plate I-9, II8). This stage was rather short, and no chromosomal aberration was observed.

Metaphase II: In most of the cells, chromosome arranged precisely on the equatorial plate and two spindles were parallel to each other (Plate I-10, II-9). However, a number of anomalies, including chromosome straggling (Plate III-7), tripolar spindle (Plate III-8), V spindle, T spindle (Plate III-9) and I spindle, were observed. Autotetraploid line, 96025, showed the highest frequency of abnormal spindles $(31.08 \%)$ during Metaphase II (Table 4). In addition, around $10.00 \%$ asynchronous division of the dyad cell was found. The frequency of abnormal chromosome behavior of newly developed line was significantly lower than its parents.

Anaphase II: The chromatids separated and move towards opposite poles (Plate I-11, II-10). The chromosome straggling was relatively low, while high frequency of asynchronous division (Plate III-10) was found in this stage. Autotetraploid rice line 96025 showed the highest number of asynchronous divisions (Table 5).

Telophase II: The chromosomes of the dyad cell reached at the two poles of each cell and condensed to reform nuclear membrane and nucleolus (Plate I-12, II-11), then the isobilateral tetrads were formed. In addition to the normal tetrads (Plate II-12), some abnormalities, linear tetrad (Plate III-11), T tetrad (Plate III-12), polyad and micronucleus cell, were found at telophase II (Table 6).

Pollen and embryo sac fertility of new line 1162 and its hybrids: Pollen fertility of new line 1162 and its hybrids was generally higher than the parents or other autotetraploid lines (Table 7). Pollen fertility of IR36-4x was $45.79 \%$, lower than other materials $(65 \%)$. While pollen fertility of $F_{1}$ hybrid (IR36-4x $\times 1162$ ) was improved to $76.45 \%$ (Table 7). Stained abortive pollens and small pollens were the main types of abortive pollens in parents and $\mathrm{F}_{1}$, respectively.

The new line 1162 and its hybrids mostly produced normal embryo sacs and fertility of embryo sac was over $80 \%$ (Table 8). Normal embryo sac contains one egg cell, two synergids at the micropylar end, two polar nuclei above the egg apparatus and a group of antipodal cells at chalazal end. 
Table 7. Pollen fertility of autotetraploid rice and hybrids.

\begin{tabular}{|c|c|c|c|c|c|c|c|}
\hline \multirow[t]{2}{*}{ Name } & \multirow[t]{2}{*}{$\mathbf{N}$} & \multirow[t]{2}{*}{ Normal/\% } & \multicolumn{3}{|c|}{ Stained abortive pollen (\%) } & \multirow{2}{*}{$\begin{array}{c}\text { Round abortive } \\
\text { pollen }(\%)\end{array}$} & \multirow{2}{*}{$\begin{array}{c}\text { Typical } \\
\text { abortive } \\
\text { pollen }(\%)\end{array}$} \\
\hline & & & $\begin{array}{c}\text { Medium } \\
\text { pollen }\end{array}$ & $\begin{array}{l}\text { Large } \\
\text { pollen }\end{array}$ & $\begin{array}{l}\text { Small } \\
\text { pollen }\end{array}$ & & \\
\hline 1162 & 192 & $91.03 \pm 3.56$ & 0 & 0 & $1.40 \pm 2.19$ & $63 \pm 1.40$ & $2.82 \pm 4.12$ \\
\hline $1162 \times$ Jing $x$ & 219 & $68.28 \pm 7.13$ & $10.19 \pm 1.57$ & 0 & $15.18 \pm 2.83$ & 0 & $0.40 \pm 0.89$ \\
\hline $1162 \times 4001-1-4 x$ & 338 & $83.39 \pm 3.96$ & 0 & 0 & $0.82 \pm 0.77$ & $0.31 \pm 0.70$ & $0.31 \pm 0.70$ \\
\hline $1162 \times$ Yuhei $1-4 x$ & 268 & $80.81 \pm 4.19$ & $3.77 \pm 0.91$ & 0 & $2.97 \pm 1.69$ & $0.60 \pm 1.19$ & $1.78 \pm 1.30$ \\
\hline $1162 \times$ IR36-4x & 387 & $76.30 \pm 6.25$ & $2.15 \pm 2.55$ & 0 & $3.67 \pm 0.82$ & $1.67 \pm 0.54$ & $4.68 \pm 2.74$ \\
\hline 96025 & 148 & $65.27 \pm 16.77$ & $21.38 \pm 9.04$ & $0.74 \pm 1.47$ & $6.34 \pm 7.62$ & $5.14 \pm 1.61$ & $2.58 \pm 3.33$ \\
\hline Jackson-4x & 167 & $70.29 \pm 7.60$ & $4.88 \pm 4.22$ & $0.76 \pm 1.31$ & $2.72 \pm 2.97$ & $0.49 \pm 0.85$ & $2.12 \pm 2.29$ \\
\hline Jingxian $89-4 x$ & 154 & $70.61 \pm 6.34$ & $17.89 \pm 5.11$ & $1.09 \pm 1.26$ & 0 & $0.57 \pm 1.14$ & $3.98 \pm 3.46$ \\
\hline Yuhei 1-4x & 78 & $74.80 \pm 5.56$ & $13.14 \pm 2.55$ & 0 & $1.75 \pm 3.04$ & $1.08 \pm 1.86$ & $1.19 \pm 2.06$ \\
\hline IR36-4x & 204 & $45.79 \pm 11.23$ & $31.11 \pm 6.39$ & $3.20 \pm 1.95$ & $6.05 \pm 3.49$ & $4.00 \pm 0.95$ & $1.12 \pm 0.97$ \\
\hline
\end{tabular}

$\mathrm{N}=$ No. of pollen observed.

Table 8. Fertility of embryo sacs in autotetraploid rice.

\begin{tabular}{|c|c|c|c|c|c|c|c|c|c|c|}
\hline Name & $\mathbf{N}$ & $\begin{array}{c}\text { Normal } \\
(\%)\end{array}$ & $\begin{array}{c}\text { Polar } \\
\text { nuclei } \\
\text { at abnormal } \\
\text { position }(\%)\end{array}$ & $\begin{array}{c}\text { Embryo sac } \\
\text { Degeneration } \\
(\%)\end{array}$ & $\begin{array}{c}\text { Female } \\
\text { germ unit } \\
\text { degeneration } \\
(\%)\end{array}$ & $\begin{array}{c}\text { Synergid } \\
\text { Degeneration } \\
(\%)\end{array}$ & $\begin{array}{c}\text { 'Double set' } \\
\text { of embryo sac } \\
(\%)\end{array}$ & $\begin{array}{c}\text { No egg } \\
\text { apparatus } \\
(\%)\end{array}$ & $\begin{array}{c}\text { No } \\
\text { polar } \\
\text { nuclei } \\
(\%)\end{array}$ & $\begin{array}{c}\text { Small } \\
\text { Embryo } \\
\text { sac } \\
(\%)\end{array}$ \\
\hline 1162 & 11 & 90.91 & 9.09 & 0.00 & 0.00 & 0.00 & 0.00 & 0.00 & 0.00 & 0.00 \\
\hline $1162 \times$ Jingxian $89-4 x$ & 66 & 81.82 & 4.55 & 3.03 & 3.03 & 0.00 & 1.52 & 1.52 & 1.52 & 3.03 \\
\hline $1162 \times 4001-1-4 x$ & 64 & 90.63 & 0.00 & 6.25 & 0.00 & 0.00 & 0.00 & 1.56 & 0.00 & 1.56 \\
\hline $1162 \times$ Yuhei1-4x & 44 & 88.64 & 2.27 & 2.27 & 0.00 & 2.27 & 0.00 & 0.00 & 0.00 & 4.55 \\
\hline $1162 \times$ IR $36-4 x$ & 58 & 94.83 & 1.72 & 0.00 & 0.00 & 0.00 & 0.00 & 0.00 & 0.00 & 3.45 \\
\hline
\end{tabular}

$\mathrm{N}=$ No. of embryo sacs observed.

Table 9. Fertility and seed setting in autotetraploid rice.

\begin{tabular}{lcclccc}
\hline Name & SS (\%) & $\begin{array}{c}\text { Pollen } \\
\text { fertility (\%) }\end{array}$ & Name & SS (\%) & $\begin{array}{c}\text { Pollen fertility } \\
(\%)\end{array}$ & $\begin{array}{c}\text { Embryo sac } \\
\text { fertility/\% }\end{array}$ \\
\hline 96025 & $38.10 \pm 16.61$ & $55.72 \pm 9.67$ & 1162 & $81.58 \pm 1.90$ & $91.02 \pm 3.56$ & 90.91 \\
Jackson-4x & $33.73 \pm 11.70$ & $72.71 \pm 9.65$ & $1162 \times$ Jingxian89-4x & $47.03 \pm 5.28$ & $68.28 \pm .13$ & 81.82 \\
Jingxian89-4x & $25.68 \pm 12.38$ & $70.27 \pm 7.00$ & $1162 \times$ T416 & $48.62 \pm 12.53$ & $80.81 \pm 4.19$ & 88.64 \\
Yuhei1-4x & $10.71 \pm 6.16$ & $71.06 \pm 8.46$ & $1162 \times$ Yuhei1-4x & $91.88 \pm 0.68$ & $83.3 \pm 3.96$ & 90.63 \\
IR36-4x & $27.93 \pm 14.67$ & $45.59 \pm 11.48$ & $1162 \times$ IR36-4x & $64.36 \pm 1.65$ & $76.30 \pm .25$ & 94.83 \\
\hline
\end{tabular}

$\mathrm{SS}=$ Seed setting.

Low percentage of abnormal embryo sacs found in all autotetraploid rice lines, which included embryo sac degeneration, embryo sac without female germ unit, egg apparatus degeneration, embryo sac without polar nuclei, embryo sac with polar nuclei at abnormal position and small embryo sac.

Pollen fertility and seed setting of autotetraploid rice hybrids were significantly higher than parents (Table 9). Seed setting of hybrids increased significantly as compared to other autotetraploid rice parents with low seed setting. The results suggested that the high fertility of 1162 is heritable.

Analysis of main agronomic characteristics and heterosis of new line 1162: Data regarding performance of 1162 evaluated during late season 2010 and early season 2011, and results are presented in Table 10. The mean of panicle length, number of grains per panicle, filled grains per panicle, 1000grain weight, grain length, grain width, grain length-width ratio were $25.04 \mathrm{~cm}, 103.38$ grains, 83.56 grains, $43.44 \mathrm{~g}$, $9.54 \mathrm{~mm}, 4.04 \mathrm{~mm}$ and 2.37 , respectively. The plant height of 1162 was high $(124.13 \mathrm{~cm})$, while the number of panicles per plant was less and only 4 panicles per plant were found. Seed setting was ranged from $70 \% \sim 89.22 \%$, and the average seed set $(81.58 \%)$ of newly developed line was in accordance with the seed set of diploid rice. Variation coefficient of most traits was less than $10 \%$ except for the number of panicles per plant, number of grains per panicle and filled grains per panicle. It showed that all the individuals of new line have almost similar morphological characteristics.

The results of heterosis of 13 agronomic characters of 1162 crossed with other 42 autotetraploid lines are listed in Table 11. Obvious heterosis was observed in most of the quantitative characters of $F_{1}$ hybrids, especially the characters related to the yield. The heterosis in 10 quantitative characters was not only positive but also attained highly significant 
Cytogenetic and fertility evaluation of new autotetraploid rice line

Table 10. Performance of main agronomic traits of the new line 1162.

\begin{tabular}{lrrcrr}
\hline Agronomic traits & Max & Min & Variation amplitude & Mean \pm SD & CV(\%) \\
\hline PH/cm & 135.00 & 110.00 & 25.00 & $124.13 \pm 2.04$ & 5.19 \\
NP & 5.00 & 2.00 & 3.00 & $4.00 \pm 0.37$ & 28.87 \\
PL/cm & 27.85 & 22.93 & 4.92 & $25.04 \pm 0.57$ & 7.17 \\
NGP & 145.00 & 70.20 & 74.80 & $103.38 \pm 8.41$ & 25.71 \\
NFGP & 106.70 & 59.00 & 47.70 & $83.56 \pm 5.91$ & 22.35 \\
SS $(\%)$ & 89.22 & 70.00 & 19.22 & $81.58 \pm 1.90$ & 7.38 \\
TGW/g & 45.71 & 41.03 & 4.68 & $43.44 \pm 0.51$ & 3.70 \\
GL/mm & 9.70 & 8.90 & 0.80 & $9.54 \pm 0.07$ & 2.48 \\
GW/mm & 4.30 & 3.60 & 0.70 & $4.04 \pm 0.07$ & 5.86 \\
L/W & 2.64 & 2.06 & 0.58 & $2.37 \pm 0.05$ & 7.27 \\
\hline
\end{tabular}

$\mathrm{CV}=$ Coefficient of Variation; See Table 1 for traits abbreviations

Table 11. Mean heterosis and transgressive heterosis of main quantitative traits.

\begin{tabular}{|c|c|c|c|c|c|c|}
\hline \multirow[b]{2}{*}{ Traits } & \multicolumn{3}{|c|}{$\left(H_{P M}\right)$ Mid-parent heterosis } & \multicolumn{3}{|c|}{$\left(H_{P B}\right)$ Better-parent heterosis } \\
\hline & Mean & $\begin{array}{c}\mathbf{H}>\mathbf{0} \\
\text { Combination }\end{array}$ & $\begin{array}{c}\mathbf{H}<0 \\
\text { Combination }\end{array}$ & Mean & $\begin{array}{c}\mathrm{H}>\mathbf{0} \\
\text { Combination }\end{array}$ & $\begin{array}{c}\mathbf{H}<0 \\
\text { Combination }\end{array}$ \\
\hline$\overline{\mathrm{PH}}$ & $0.309^{* *}$ & $41(37)$ & $1(0)$ & 0.244 & $41(19)$ & $1(0)$ \\
\hline NP & -0.026 & $20(6)$ & $22(7)$ & $-0.260 *$ & $16(1)$ & $26(19)$ \\
\hline PL & $0.151^{* *}$ & $36(23)$ & $6(0)$ & 0.111 & $33(17)$ & $9(9)$ \\
\hline NGP & $0.473 * *$ & $40(28)$ & $2(0)$ & 0.376 & $38(11)$ & $4(4)$ \\
\hline NFGP & $0.834^{* *}$ & $41(29)$ & $1(0)$ & 0.513 & $37(12)$ & $5(5)$ \\
\hline SS & $0.429 * *$ & $39(33)$ & $3(0)$ & 0.001 & $26(6)$ & $16(13)$ \\
\hline TGW & -0.032 & $18(10)$ & $24(12)$ & $-0.218 * *$ & 0 & $42(34)$ \\
\hline SWP & 0.690 ** & $40(22)$ & $2(0)$ & 0.119 & $21(2)$ & $21(16)$ \\
\hline GWP & $0.797 * *$ & $39(28)$ & $3(0)$ & 0.724 & $38(22)$ & $4(4)$ \\
\hline GWP/SWP & $0.155^{* *}$ & $27(12)$ & $15(8)$ & $-0.408 * *$ & $7(0)$ & $35(28)$ \\
\hline GL & $0.062^{* *}$ & $35(24)$ & $7(2)$ & $-0.020 * *$ & 21(8) & 21(16) \\
\hline GW & $0.103 * *$ & $40(24)$ & $2(0)$ & 0.038 & $31(2)$ & $11(6)$ \\
\hline GL/GW & $-0.048 * *$ & $10(5)$ & $32(8)$ & $-0.146 * *$ & $4(0)$ & $38(31)$ \\
\hline
\end{tabular}

$\mathrm{PH}=$ Plant Height, NP=Number of Panicles, $\mathrm{PL}=$ Panicle Length, NGP= Number of Grains per Plant, NFGP= Number of Filled Grains per Panicle, SS=Seed Setting, TGW=1000-Grains Weight, SWP=Straw Weight per Plant, GWP=Grain Weight per Plant, GWP/SWP= Ratio of GWP to SWP, GL=Grain Length, GW=Grain Width, GL/GW=Grain LengthWidth Ratio

${ }^{*},{ }^{* *}$ Significantly different from zero at $\mathrm{p}<0.05$ and $\mathrm{p}<0.01$, respectively. The values in parenthesis represent the number of combinations with significantly different from zero at $\mathrm{p}<0.05$

level. The mid-parent heterosis of these 10 quantitative characters was in sequence of number of filled grains per panicle>grain weight per plant>straw weight per plant $>$ number of grains per plant $>$ seed setting $>$ plant height $>$ grain-straw ratio>panicle length $>$ grain width $>$ grain length. The mid-parent heterosis of other remaining three quantitative characters was negative. The population midparent heterosis for number of filled grains per panicle in 41 cross sets was significant, and 29 cross sets showed positive heterosis, while one cross set showed negative heterosis. A higher mid-parent heterosis value was also found for grain weight per plant, straw weight per plant, number of grains per plant, seed setting and plant height. In total, more than 38 cross sets showed positive heterosis, and 20 cross sets attained significant level. Heterosis for seed setting in 39 cross sets was positive, 33 cross sets showed significant positive heterosis, while three cross sets showed negative heterosis. The results showed that the mid-parent heterosis for most of the quantitative characters of 1162 was significant as compared to other autotetraploid lines.

Better-parent heterosis values of 8 quantitative characters, including plant height, panicle length, number of grains per panicle, number of filled grains per panicle, seed setting, straw weight, grains weight per plant and grain width, was positive, but didn't reach the remarkable level. Grain weight per plant showed the maximum better-parent heterosis value (0.724) and number of filled grains per plant ranked second (0.513). A total of 38 and 37 cross sets expressed positive heterosis for grain weight and number of filled grains per plant, respectively. Better-parent heterosis value of seed 
setting was 0.001 , positive heterosis of 6 cross sets was significant. These results indicate that mid-parent heterosis and better-parent heterosis for most of the quantitative characters of the hybrids crossed with 1162 have the significant population mid-parent heterosis and better-parent heterosis, and have a wide heterosis, especially for grain weight per plant, filled grains per plant and seed setting. Most of the cross sets for these characters showed the positive heterosis. In addition, it is worthwhile to mention that heterosis for seed setting was significant among most of the cross sets.

\section{DISCUSSION}

Low trivalent and univalent might be the main reason for high fertility in newly developed autotetraploid rice: Abnormal meiosis chromosome behavior, such as multivalents and univalents, happened frequently in autotetraploid plants, and was considered important reasons for low seed setting of the autotetraploids (Luan et al., 2007; Long et al., 2007; He et al., 2011a,b). Multivalents of chromosome included trivalents, quadrivalents, pentavalents, and so on, in autotetraploid plants. There are two viewpoints about the influence of quadrivalent on seed setting of autotetraploid plants. The first one pointed out that the more number of quadrivalents cause reduction in seed setting of autotetraploid plants (Sebastiampillai et al., 1977). Another study on chromosome configuration of autotetraploid rice and its hybrids, revealed that ring quadrivalents and number of quadrivalents had negative correlation with pollen fertility and seed setting, respectively (He et al., 2011b). He et al. (2011a) reported higher number of multivalent, trivalent and univalent in autotetraploid rice that lead to low seed in autotetraploid rice hybrids. The second viewpoint considered that the frequency of quadrivalents was not the crucial factor that influences the seed setting. The numbers of quadrivalents were approximately similar among the hybrids and their parents with different pollen fertility (Zhao et al., 2006). Luan et al. (2007) studied the cytology of the autotetraploid restorer lines and found that univalents, trivalents and multivalents result in low seed setting, while the quadrivalent had nonsignificant correlation with seed setting. They considered that the number of quadrivalents within a range may not exert great effect on pollen fertility or seed setting. Long et al. (2007) considered that the autotetraploid rice maintainer lines with more number of quadrivalents exhibited more stable inheritance than the autotetraploid rice restorer lines, and believed that the increase in quadrivalents results in good chromosome pairing of maintainer lines. High percentage of trivalents and univalent were generally resulted in low seed setting of autotetraploid plants (Cai et al., 2007; Luan et al., 2007; He et al., 2011a,b; Wu et al., 2014).

In our study, more number of quadrivalents were found in newly developed autotetraploid rice 1162 than that in its parents with low seed setting, while the frequency of trivalent and univalent was lower in the new line comparing with its parents at diakinesis and metaphase I. Moreover, the configuration of chromosome in 1162 was symmetrical, which may be in favor of $2 / 2$ separation of homologous chromosomes. These results suggested that high quadrivalents and low trivalent and univalent improve the hereditary stability of the new line 1162 than its parents, and it might be the important factor for high fertility of new autotetraploid line.

Newly developed autotetraploid line 1162 is a new germplasm that could be used for the breeding of autotetraploid rice: High fertility autotetraploid rice is a key germplasm for autotetraploid rice breeding. As a result, rice breeders kept on developing high fertility autotetraploid rice lines for more than 60 years. Till to nineties in twentieth century, some autotetraploid rice hybrids with high seed setting were developed. Two autotetraploid rice lines, PMeS1 and PMeS-2, with more than 65\% seed setting were produced (Cai et al., 2007). Luan et al. (2008) reported two autotetraploid rice restorers with high seed set. These studies give a hope to autotetraploid rice breeders for commercial production. Here, we developed a new autotetraploid rice line with high fertility that could be used to generate autotetraploid hybrids with high fertility.

Conclusion: In present study, we reported a newly developed line (1162) that showed very high pollen and embryo sac fertility, and high seed setting $(>80 \%)$. Further, newly developed line showed high hybrid vigor and better agronomic traits than parents. Substantial evidences proved that the new line had the potential to improve the seed set of autotetraploid lines and had great potential to increase the yield of autotetraploid rice because of its gigantic features, such as long and wide grains, long panicle and high seed setting. Therefore, further studies on the new line have significant implications for genetic improvement of autotetraploid rice. This research may promote autotetraploid rice breeding to a new level.

Acknowledgements: The authors thank Ms. Shuhong Yu and other lab mates for assistance in development of a new autotetraploid line 1162. This work was supported by the NSFC (31571625), the open project of State Key Laboratory for Conservation and Utilization of Subtropical Agrobioresources (2015), Guangdong Provincial Science and Technology Project (2014A030304055) and Guangdong provincial key platform of University and major research project (Natural science) - characteristic innovation project [Yue-JK2014(65)].

\section{REFERENCES}

Cai, D.T., L.P. Yuan and X.G. Lu. 2001. A new strategy of 
rice breeding in $21^{\text {st }}$ century II. Searching a new pathway of rice breeding by utilization of double heterosis of wide cross and polyploidization. Acta Agron. Sin. 27:110-116.

Cai, D.T., J.G. Chen, D.L. Chen, B.C. Dai, W. Zhang, Z.J. song, Z.F. Yang, C.Q. Du, Z.Q. Tang, Y.C. He, D.S. Zhang, G.C. He and Y.G. Zhu. 2007. Development of two polyploid rice lines with meiosis stability. Sci. China Life Sci. 37:217-226.

Guo, H.B., X.D. Liu, Y.G. Lu and J.H. Feng. 2006. Structure of mature embryo sac and its abnormal phenomena in autotetraploid rice. Chinese J. Rice Sci. 20:283-289.

He, J.H., M.Q. Shahid, Z.X. Chen, X.A. Cheng, X.D. Liu and Y.G. Lu. 2011a. Abnormal PMC microtubule distribution pattern and chromosome behavior. Plant Syst. Evol. 291:257-265.

He, J.H., M.Q. Shahid, Y.J. Li, H.B. Guo, X.A. Cheng, X.D. Liu and Y.G. Lu. 2011b. Allelic interaction of $F_{1}$ pollen sterility loci and abnormal chromosome behavior caused pollen sterility in intersubspecific autotetraploid rice hybrids. J. Exp. Bot. 62:4433-4445.

Hu, C.Y., Y.X. Zeng, H.B. Guo, Y.G. Lu, Z.X. Chen, M.Q. Shahid and X.D. Liu. 2010. Megasporogenesis and megagametogenesis in autotetraploid indica/japonica rice hybrid. Rice Sci. 17:296-302.

Huang, Q.C., J.S. Sun and S.L. Bai. 1999. Study on reproductive characters of autotetraploid rice. Sci. Agri. Sin. 32:14-17.

Li, X., M.Q. Shahid, J.W. Wu, L. Wang, X.D. Liu and Y.G. Lu. 2016. Comparative small RNA analysis of pollen development in autotetraploid and diploid rice. Int. J. Mol. Sci. 17:499.

Long, W.B., L. Luan, X. Wang, Y.H. Liu, S.B. Tu, F.L. Kong and T. He. 2007. Cytogenetical comparison of restorers TP-4 and D minghui63 and maintainer D46B of autotetraploid rice. Hereditas 4:462-470.

Luan, L., W.B. Long, X. Wang, Y. Chen, Y.H. Liu, S.B. Tu, F.L. Kong and W.M. Li. 2008. Agronomic traits and cytogenetic behaviors of autotetraploid rice restorers with high seed set. Chinese J. Appl. Environ. Biol. 14:1117.

Luan, L., S.B. Tu, W.B. Long, X. Wang, Y.H. Liu, F.L. Kong, W.G. Yan and M.Q. Yu. 2007. Cytogenetic studies on two $F_{1}$ hybrids of autotetraploid rice varieties showing extremely high level of heterosis. Plant Syst. Evol. 267:205-213.

Sebastiampillai, A.R. and J.K. Jones. 1977. Cytological studies in the genus Fragaria (Rosaceae) I. Chromosome association and fertility in induced autotetraploids. Cytologia 42:525-534.

Shahid, M.Q., F.Y. Chen, H.Y. Li, S.Z. Wang, P.F. Chen, S.Q. Lin, X.D. Liu and Y.G. Lu. 2013b. Double-neutral genes $S_{a}{ }^{n}$ and $S_{b}{ }^{n}$, for pollen fertility in rice to overcome indica $\times$ japonica hybrid sterility. Crop Sci. 53:164-176.
Shahid, M.Q., G.F. Liu, J.Q. Li, M. Naeem and X.D. Liu. 2011. Heterosis and gene action study of agronomic traits in diploid and autotetraploid rice. Acta Agri. Scand. B-S P. 61:23-32.

Shahid, M.Q., J.F. Sun, C.M. Wei, P. Zhang and X.D. Liu. 2010. Studies on the abnormality of embryo sac and pollen fertility in autotetraploid rice during different growing seasons. Pak. J. Bot. 42:7-19.

Shahid, M.Q., H.M. Xu, S.Q. Lin, Z.X. Chen, M. Naeem, Y.J. $\mathrm{Li}$ and X.D. Liu. 2012. Genetic analysis and hybrid vigor study of grain yield and other quantitative traits in autotetraploid rice. Pak. J. Bot. 44:237-246.

Shahid, M.Q., Y.J. Li, M.F. Saleem, M. Naeem, C.M. Wei and X.D. Liu. 2013a. Yield and yield components in autotetraploid and diploid rice genotypes (indica and japonica) sown in early and late seasons. Aust. J. Crop Sci. 7:632-641.

Song, W.C. and Y.H. Zhang. 1992. Rice tetraploidy and its effect on agronomic traits and nutritional constituents. Acta Agron. Sin. 2:137-144.

Tu, S.B., L. Luan, Y.H. Liu, W.B. Long, F.L. Kong, T. He, Q.F. Xu, W.G. Yan and M.Q. Yu. 2007. Production and heterosis analysis of rice autotetraploid hybrids. Crop Sci. 47:2356-2363.

Wu, J.W., C.Y. Hu, M.Q. Shahid, H.B. Guo, Y.X. Zeng, X.D. Liu and Y.G. Lu. 2013. Analysis on genetic diversification and heterosis in autotetraploid rice. Springer Plus 2:1-12.

Wu, J.W., M.Q. Shahid, H.B. Guo, W. Yin, Z.X. Chen, L. Wang, X.D. Liu and Y.G. Lu. 2014. Comparative cytological and transcriptomic analysis of pollen development in autotetraploid and diploid rice. Plant Reprod. 27:181-196.

Wu, J.W., M.Q. Shahid, L. Chen, Z.X. Chen, L. Wang, X.D. Liu and Y.G. Lu. 2015. Polyploidy enhances $F_{1}$ pollen sterility loci interactions that increase meiosis abnormalities and pollen sterility in autotetraploid rice. Plant Physiol. 169:2700-2717.

Zhang, H.H., J.H. Feng, Y.G. Lu, B.Y. Yang and X.D. Liu. 2003. Observation on formation and development of autotetraploid rice embryo sac using laser scanning confocal microscope. J. Chinese Electron Micro. Soc. 22:380-384.

Zhao, M.H., X.D. Liu, Y.G. Lu, J.Q. Li and H.B. Guo. 2006. Chromosome pairing behavior and reproduction in the hybrid developed by the interaction of different pollen sterile genes in autotetraploid rice. Acta Agron. Sin. 32:1472-1478. 